04

\title{
Влияние плотности плазмы на степень подавления сигналов аналитов в ICP-MS
}

\author{
() Т.К. Нурубейли
}

Институт фризики НАН Азербайджана, Az-1143 Баку, Азербайджан

e-mail: kamilnuri@rambler.ru

Поступило в Редакцию 7 февраля 2020 г.

В окончательной редакции 3 марта 2020 г.

Принято к публикации 16 июня 2020 г.

Рассмотрены возможные влияния ионизационных явлений на аналитический сигнал в методе масс-спектрометрии с индуктивно связанной плазмой с высокотемпературным источником ионов. Рассчитаны и экспериментально определены зависимости степени подавления ионных сигналов от концентрации электронов для ионов матричных и приместных элементов. Показано, что степень подавления сигналов достаточно сильно зависит от концентрации электронов в аналитической зоне в присутствии матричных элементов с различными по величине первыми потенциалами ионизации. На примере масс-спектрометра ICP-MS (Agilent Technologies) изучены влияния мощности индуктивной плазмы и скорости распылительного аргона на чувствительность масс-спектрометра. Результаты эксперимента показали, что причины подавления сигналов аналитов на выходе, по-видимому, связаны с ионно-электронной рекомбинацией ионов (как матричных, так и примесных) в области плазмы.

Ключевые слова: масс-спектрометрия с индуктивно связанной плазмой, матричный эффект, рекомбинация ионов, концентрация электронов, подавление ионных сигналов.

DOI: $10.21883 / J T F .2020 .12 .50121 .46-20$

\section{Введение}

Метод масс-спектрометрии с индуктивно связанной плазмой (ICP-MS) в настоящее время является одним из основных многоэлементных методов анализа различных веществ. Уже через несколько лет после введения ICP-MS в аналитическую практику было установлено, что метод имеет ограничения, связанные со спектральными и неспектральными матричными эффектами [1], которые уменьшают количество определяемых элементов и увеличивают их предел обнаружения.

Под спектральными интерференциями понимают взаимное наложение в масс- спектре сигналов различных ионов с примерно одинаковым значением отношения массы к заряду $(m / q)$. За годы развития ICP-MS достигнут значительный прогресс в устранении спектральных наложений [2]. Значительно хуже обстоят дела с устранением неспектрального матричного эффекта, под которым понимается ряд явлений, приводящих к подавлению (усилению) сигнала аналита в присутствии матричного элемента (элементов растворителя или внутреннего стандарта). Причины проявления матричного эффекта к настоящему времени достаточно хорошо изучены, предложен ряд способов его учета и снижения [3]. Однако следует отметить, что, несмотря на тридцать лет развития ICP-MS, в методе по прежнему действуют ограничения по содержанию матрицы в анализируемом растворе на уровне $0.05-0.2$ mass.\% в зависимости от природы матричного элемента. Известными причинами таких явлений являются сильная ионизация многих элементов периодической системы, а также изменение эффективности транспортировки ионов аналитов за счет сильных полей пространственного заряда в ионном пучке [4].

Большинство встречающихся в литературе экспериментальных данных о матричных ионизационных явлениях в ICP-MS представляют собой чаще всего отрывочные сведения о степени подавления $\gamma\left(A_{n}\right)_{M_{t}}$ ионного сигнала $A_{n}$ для одного, реже нескольких, определяемых элементов и одного матричного $M_{t}$, как правило, легкоионизируемого. За степень подавления ионного сигнала $\gamma\left(A_{n}\right)_{M_{t}}$ принимали соотношение его сигналов в присутствии (нижний индекс $-M_{t}$ ) и в отсутствии $\left(\mathrm{H}_{2} \mathrm{O}\right)$ матричных элементов [5]:

$$
\gamma\left(A_{n}\right)_{M_{t}}=S\left(A_{n}^{+}\right)_{M_{t}} / S\left(A_{n}^{+}\right)_{\mathrm{H}_{2} \mathrm{O}}
$$

Вообще подавление сигналов аналитов объясняется наличием в составе матричных элементов (калибровочных и внутренних стандартов) атомов с первым потенциалом ионизации $\varphi_{i}^{+}<4.33-8.33 \mathrm{eV}$ [5], т. е. легкоионизируемых элементов. В настоящее время считается, что эффект подавления ионного сигнала в методе ICP-MS увеличивается прямо пропорционально массе матричного иона [4].

На основании литературных данных можно сделать следующие выводы. Наиболее сильные ионизационные влияния в ICP-MS оказывают легкоионизируемые элементы при их концентрации в анализируемом растворе выше 0.01-0.1 М. Матричные элементы с высоким потенциалом ионизации также могут подавлять ионизацию 
примесных элементов, если присутствуют в достаточном количестве [6,7].

Не прослеживается отчетливой связи потенциала ионизации атомов определяемого элемента со степенью ионизационных влияний. В работе [8] даже утверждается, что значение $\gamma\left(A_{n}\right)_{M_{t}}$ связано с массой определяемого иона, в то время как взаимосвязь с $\varphi_{i}^{+}$не проявляется. Степень потери аналита в процессе транспортировки ионного пучка, вероятно, обратно пропорционально массе ионов аналита, и вызывает более серьезные воздействия при меньших массах.

Настоящая работа посвящена рассмотрению влияния ионизационных процессов в плазме индуктивно связанного разряда на подавление аналитических сигналов в методе ICP-MS. При этом следует иметь в виду, что центральная (аналитическая) зона находится на оси индуктивного разряда, в которую вводится пробосодержащий аэрозоль, и из нее идет отбор ионов, при этом аналитическая зона находится в состоянии термодинамического равновесия.

\section{1. Теоретическая часть}

Наиболее серьезная попытка теоретического осмысления подавления аналитического ионного сигнала различными матричными солями в методе ICP-MS за счет ионизационных эффектов была предпринята в работе [7]. Авторы принимали в расчетах, что электронная плотность в ICP-MS обеспечивается ионизацией аргона и атомных компонентов гомогенных солей $(\mathrm{NCl}, \mathrm{MgCl}$, $\mathrm{NH}_{4} \mathrm{~J}, \mathrm{NH}_{4} \mathrm{Cl}$ ), вводимых с водным аэрозолем анализируемого раствора в различных концентрациях в аргоновую плазму. Расчеты по константам $\varphi_{i}^{+}$в предположении существования локального термодинамического равновесия (ЛТР) для ионизационной температуры $7500 \mathrm{~K}$ независимой от концентрации матричной соли показали, что теоретические значения количества матричной соли, обеспечивающие заметные влияния на сигнал аналита, должны быть на 1-2 порядка выше, чем экспериментальные значения. Причина столь существенных расхождений экспериментальных и теоретических результатов авторами объясняется изменением температуры ионизации в процессе экстракции ионов при введении высоких концентраций матричных элементов.

Ощутимые матричные ионизационные влияния в ICP, наблюдаемые при введении легкоионизируемых элементов различной атомной массы, свидетельствуют о ЛТР в аналитической зоне, проявляющимся в смещении влево реакции ионизации атомов определяемого элемента за счет ион-электронной рекомбинации

$$
A_{n} \rightleftarrows A_{n}^{+}+e
$$

Следовательно, можно рассматривать константу равновесия реакции первичной ионизации атомов $K_{T}\left(A_{n}\right)$, исходя из уравнения Саха

$$
\begin{aligned}
K_{T}\left(A_{n}\right)= & \frac{N(e) N\left(A_{n}^{+}\right)}{N\left(A_{n}\right)}=\frac{[2 \pi m(e)]^{\frac{3}{2}} 2 Z_{T}\left(A_{n}^{+}\right)}{h^{2} Z_{T}\left(A_{n}\right)} \\
& \times \exp \left[\frac{e \varphi^{+}\left(A_{n}\right)}{k T}\right],
\end{aligned}
$$

где $N(e), N\left(A_{n}^{+}\right)$и $N\left(A_{n}\right)$ - концентрации электронов, ионов и атомов аналитов в плазме разряда; $m(e)-$ масса электрона; $k$ - постоянная Больцмана; $h-$ постоянная Планка; $Z_{T}\left(A_{n}^{+}\right)$и $Z_{T}\left(A_{n}\right)-$ суммы по состояниям для иона и атома при температуре $T$. Можно сказать, что $\gamma\left(A_{n}^{+}\right)_{M_{t}}$ представляет собой многопараметрическую функцию, поскольку степень ионизации атомов определяется в основном $\varphi_{i}\left(A_{n}\right), T, N(e)$ и некоторое значение имеет соотношение $Z_{T}\left(A_{n}^{+}\right) / Z\left(A_{n}\right)$. Величины $\varphi_{i}^{+}\left(A_{n}\right)$ и $Z_{T}\left(A_{n}^{+}\right) / Z_{T}\left(A_{n}\right)$ являются справочными, и их воздействие на степень ионизации элемента легко рассчитывается. Температура разряда зависит от плазмообразующего газа, его расхода, конструкции горелки, рассматриваемой зоны, количества вводимого в плазму аэрозоля, анализируемого раствора, вкладываемой мощности и частоты ВЧ энергий [9]. Наиболее сложно проследить влияние $N(e)$ на значение $\gamma\left(A_{n}\right)_{M_{t}}$, поскольку концентрация электронов зависит от множества экспериментальных факторов и существенно изменяется при введении матричного элемента.

В отсутствие матричных элементов электронная плотность в ICP при конкретной температуре разряда определяется степенью ионизации аргона, а также атомных и молекулярных частиц, образующихся из молекул воды. Концентрация определяемого элемента (аналита) обычно мала, и вклад, вносимый аналитом в общую электронную плотность ионизации, весьма низок [9]. При поступлении в плазму достаточно больших концентраций элементов с более низкими потенциалами ионизации, чем у аргона, кислорода и водорода, изменяется эффективный ионизационный потенциал системы и увеличивается концентрация электронов. Таким образом, значение $N(e)$ в существенной мере определяется температурой разряда, потенциалом ионизации матричных элементов и исходным соотношением концентраций аргона, воды и матричных элементов в плазме разряда.

С целью определения степени влияния различных элементов на концентрацию электронов в аналитической зоне ICP рассчитаны равновесные составы плазмы при температуре $7500 \mathrm{~K}$ для более чем 30 матричных элементов с разными значениями $\varphi_{i}^{+}$. Температура выбрана в соответствие со средним уровнем экспериментально зарегистрированных температур, обычно принимаемых в расчетах квазиравновесной модели ICP. Расчет производился для исходной смеси, соответствующей среднему значению молярного соотношения паров воды и аргона $M\left(\mathrm{H}_{2} \mathrm{O}\right) / M(\mathrm{Ar})$.

На рис. 1 представлена зависимость степени ионизации атомов $\delta(M e)$ от первого потенциала ионизации эле- 


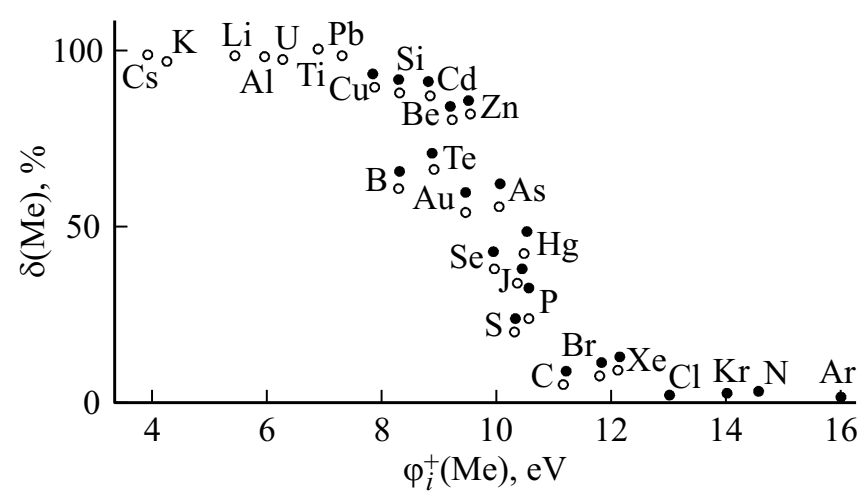

Рис. 1. Зависимость степени однократной ионизации $\delta(M e)$ от первого потенциала ионизации элементов $\varphi_{i}^{+}(M e)$ при температуре $T_{e}=7500 \mathrm{~K}$ и концентрации электронов $n_{e}=10^{15} \mathrm{~cm}^{-3}$.

ментов. Здесь можно вновь, как и для зависимости однократной ионизации, выделить по эффективности ионизационного влияния три группы элементов [10]. Легкоионизируемые $\left(\varphi_{i}^{+}(M e)<8 \mathrm{eV}, \delta(M e)>90 \%\right)$, среднеионизируемые $\left(\varphi_{i}^{+}(M e)=8-12.2 \mathrm{eV}, \delta(M e)>10-20 \%\right)$ и трудноионизируемые $\left(\varphi_{i}^{+}(M e)>12.2 \mathrm{eV}, \delta(M e)>10 \%\right)$.

Разброс точек на рис. 1 вызван тем, что степень ионизации матричного элемента, а следовательно, и концентрация электронов в ICP зависят не только от его потенциала ионизации, но и от соотношения значений сумм по состояниям иона и атома. Наибольшие различия в соотношениях $N(e)_{M_{t}} / N(e)_{\mathrm{H}_{2} \mathrm{O}}$, вызванные вариацией значений сумм по состояниям $Z_{T}\left(M_{t}^{+}\right)$и $Z_{T}\left(M_{t}\right)$, достигают $10 \%$ и наблюдаются для среднеионизируемых элементов $\left(\varphi_{i}^{+}=8-12.5 \mathrm{eV}\right)$.

Расчеты показывают (это отмечено также в [10]), что на концентрацию электронов в ICP значительно влияет молярное соотношение паров воды и аргона $M\left(\mathrm{H}_{2} \mathrm{O}\right) / M(\mathrm{Ar})$. Это выражается в том, что при уменьшении концентрации паров воды в аргоне в пять раз концентрация электронов в плазме разряда в присутствии легкоионизируемых элементов растет практически вдвое. Таким образом, при осушении аэрозоля должны возрастать ионизационные влияния в методе ICP, т.е. увеличиваться подавление сигнала аналита.

С использованием уравнения Саха (2) рассчитана степень однократной ионизации многих элементов при фиксированных параметрах плазмы $(T=7500 \mathrm{~K}$, $\left.N(e)=10^{15} \mathrm{~cm}^{-3}\right)$, и сделан вывод, что для большинства из 54 имеющихся у нас элементов степень ионизации должна превышать 90\%. Эти данные по степени ионизации различных элементов (особенно трудноионизируемых) в литературе практически отсутствуют. Максимальное содержание дважды заряженных ионов, согласно оценкам по уравнению Саха (расчет проведен для $\mathrm{La}, \mathrm{Pr}, \mathrm{Ba}, \mathrm{Yb}, \mathrm{Gd}$ ) составляет 7-10\%.

Проведенные расчеты показали, что при одинаковых температурах равновесная концентрация электронов в многокомпонентной системе, находящейся в термоди- намическом равновесии, должна быть даже ниже, чем обычно принимаемая для системы аргон-аналит, находящейся в условиях ЛТР, например, $N(e)=10^{15} \mathrm{~cm}^{-3}$ для $T=7500 \mathrm{~K}$. Согласно нашим расчетам, концентрация электронов может достигать наиболее часто приводимого в литературе уровня $N(e) \geq(1-3) \cdot 10^{15} \mathrm{~cm}^{-3}$, подтвержденного современными измерениями лазерного, томпсоновского и релеевского рассеяния [11] только при температурах выше $7500 \mathrm{~K}$.

Таким образом, согласно расчетам, эффективность однократной ионизации большой группы (свыше 70\%) легкоионизируемых элементов должна обеспечивать при равных условиях их близкие молярные пределы обнаружения в методе ICP-MS, в то время как для среднеи трудноионизируемых элементов пределы обнаружения в первую очередь должны определяться значениями $\delta\left(M_{e}\right)$ (рис. 1). Это подтверждалось в предыдущих экспериментах [12].

\section{2. Экспериментальная часть}

\section{1. Аппаратура}

Масс-спектрометр с индуктивно связанной плазмой Agilent Technologies работает при следующих рабочих параметрах: выходная мощность высокочастотного генератора $1400 \mathrm{~W}$, расход плазмообразующего газа $121 / \mathrm{min}$, вспомогательного - 0.91/min. При всех настройках прибора уровень оксидных ионов $\mathrm{CeO}^{+} / \mathrm{Ce}^{+}$ был меньше $2.5 \%$, а уровень двухзарядных ионов $\mathrm{Ce}^{++} / \mathrm{Ce}^{+}-$меньше $1 \%$. Для определения величины подавления оксидного сигнала, вызванного матричными элементами, использовали метод, предлагаемый в [13].

\section{2. Реактивы}

В качестве растворителя использовали $\mathrm{HNO}_{3}$ (Suprapur производства Merck Германия); деонизированную воду с удельным сопротивлением не менее $18.0 \mathrm{M} \Omega \cdot \mathrm{cm}$; многоэлементный стандартный раствор „ICP-MS Multielement Standard A“, содержащий Sb, As, Be, Cd, Ca, Cr, Co, Cu, Fe, Pb, Li, Mg,Mo, Ni, Se, Sr, Tl, $\mathrm{Ti}, \mathrm{Zn}, \mathrm{U}$, а также одноэлементный стандартный $\mathrm{Mn}, \mathrm{Cu}$, In, La, и Pb производства Higt-Puririty Standard (США).

Для определения роли матричного эффекта были подготовлены три типа растворов: многоэлементный стандартный раствор „Standard A“, содержащий $10 \mu \mathrm{g} / 1$ каждого элемента (тип № 1); растворы матричного элемента в составе In, Hf и $\mathrm{Pb}$, (от 0.05 до $0.5 \mathrm{~g} / \mathrm{l}$ ), $\mathrm{Cu}$, $\mathrm{La}$, и $\mathrm{Mn}(0.05-2 \mathrm{~g} / \mathrm{l})$ с добавкой „Standard $\mathrm{A}^{\text {“ }}$ (тип № 2), а также без добавки „Standard A“ (тип № 3 ).

Величину матричного эффекта подавления $X$ рассчитывали как

$$
X=\left(J_{i}-J_{i, b c k g r}\right) / J_{i, o},
$$

где $J_{i}-$ интенсивность сигнала изотопа ,i“ примесного элемента в растворе матричного элемента с 
добавкой „Standard $\mathrm{A}^{\text {“; }} J_{i, b c k g r}$ - интенсивность сигнала изотопа „“ примесного элемента без добавки; $J_{i, o}$ - интенсивность сигнала изотопа „““ в раствоpe без матричного элемента. Для большинства изотопов величина $J_{i, b c k g r} \ll J_{i}$, и можно считать, что $X=\left(J_{i}-J_{i, b c k g r}\right) / J_{i, o} \approx J_{i} / J_{i, o}$. Таким образом, определение подавления аналитического сигнала на выходе анализатора сводится к измерению соотношения интенсивностей сигнала выбранных аналитов в массовом спектре.

Перед выполнением анализов в ICP-MS проводилась настройка параметров работы масс-спектрометра на максимальную чувствительность при минимальном уровне оксидных и двухзарядных ионов путем подбора оптимальных значений расхода образца, распылительного газа, вспомогательного потока аргона, положения горелки по отношению к конусам интерфейса, мощности горелки и ионной оптики.

\section{3. Метод измерения концентрации электронов}

Для измерения концентрации электронов и температуры плазмы использовался монохроматор МДР23ЛОМО [14]. В плоскости входной щели монохроматора с помощью линзы строилось изображение излучающего объекта (аргоновой плазмы при атмосферном давлении). Входная щель монохроматора имела прямоугольную форму, а ее ширина составила $\sim 10 \mu \mathrm{m}$. В зависимости от положения линзы имелась возможность измерения электронной плотности в различных сечениях разрядного промежутка. На месте выходной щели монохроматора располагалась CCD-камера PI-MAX (Princeton Instruments), с помощью которой регистрировался оптический сигнал.

Для определения концентрации электронов в плазме индукционного разряда был выбран метод, основанный на измерении уширения спектральной линии вследствие эффекта Штарка. В диапазоне электронных концентраций $10^{14}-10^{18} \mathrm{~cm}^{-3}$ точность данного метода составляет $\sim 30 \%$.

Значения электронной концентрации вычислялись по формуле [14]:

$$
\Delta \lambda_{1 / 2}=8.6 \cdot 10^{-19} \lambda_{0}^{2} N(e)^{2 / 3}\left(1-0.7 N_{D}^{-\frac{1}{2}}\right) \cdot\left(\frac{Z_{p}^{3 / 2}}{Z_{e}}\right)
$$

Здесь $\Delta \lambda_{1 / 2}$ - полуширина штарковски уширенной спектральной линии; $N(e)$ - концентрация электронов, $\left[\mathrm{cm}^{-3}\right] ; N_{D}=4 / 3 \pi L_{D} \cdot N(e)$ - число частиц в сфере радиуса Дебая $L_{D}$ (зависит от температуры электронов); $Z_{e}$ - ядерный заряд излучающей частицы; $Z_{p}-$ кратность заряда ионов; $\lambda_{0}$ - длина волны $(\AA)$, соответствующая центру спектральной линии.

\section{3. Результаты и их обсуждение}

На рис. 2 представлена зависимость чувствительности масс-спектрометра от потенциала на экстракторе для различных ионов примесных элементов. Здесь представлены только 6 элементов из 35, чтобы, с одной стороны, охватить более или менее широкий диапазон значений степени подавлений, с другой - отсутствовали спектральные помехи. Видно, что чувствительность спектрометра при увеличении отрицательного потенциала на экстракторе от -100 до $-200 \mathrm{~V}$ уменьшается в среднем в 2 раза, а дальнейшее увеличение потенциала до $-300 \mathrm{~V}$ уменьшает чувствительность примерно в 4 раза для легких ионов $(\mathrm{Be}, \mathrm{Li}, \mathrm{Mn})$ и в 2.5 раза для тяжелых ионов (Th, Tb, La). При этом уровень оксидных и двухзарядных ионов не менялся, так как настройки системы ввода образца и плазменного блока оставались неизменными.

Причиной понижения чувствительности с повышением отрицательного потенциала экстракта, по видимому, является увеличение энергии ионов, которое может изменить амплитуду и форму массового пика, а это, в свою очередь, может вызвать изменение результатов измерений.

Отсутствие строгой зависимости подавления сигнала от величины атомной массы примесного элемента и

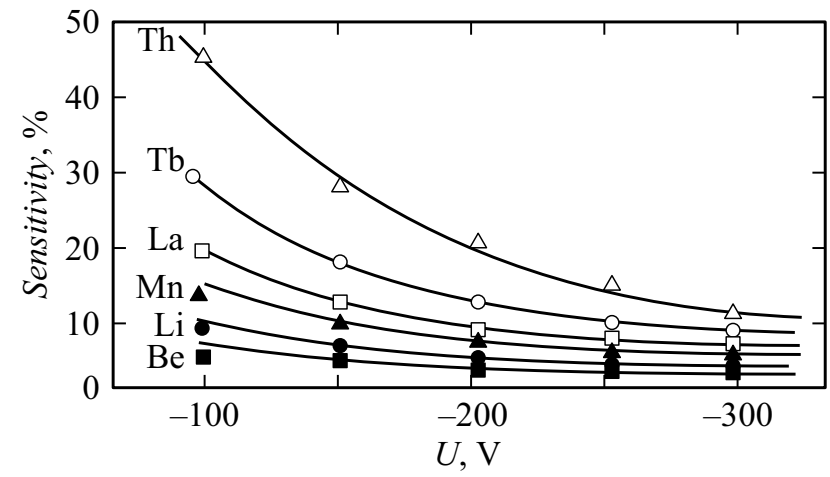

Рис. 2. Зависимость чувствительности масс-спектра от потенциала экстрактора для различных ионов примесных элементов.

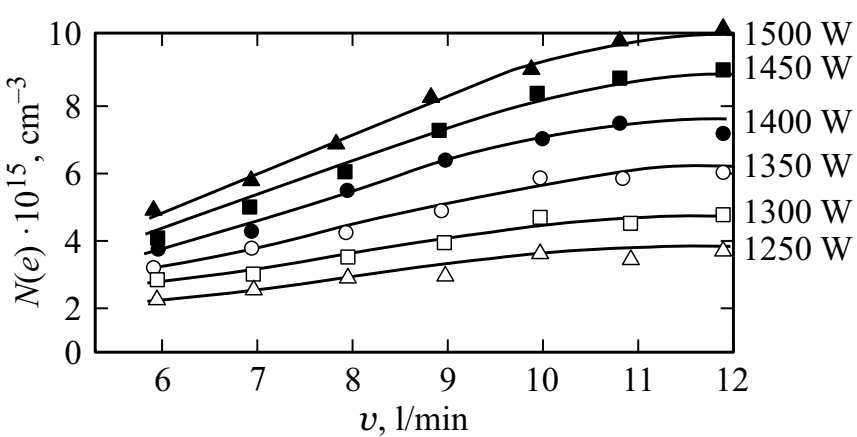

Рис. 3. Зависимость предельного значения концентрации электронов $N(e)$ в плазме от скорости аргона для разных мощностей ВЧ энергии. 


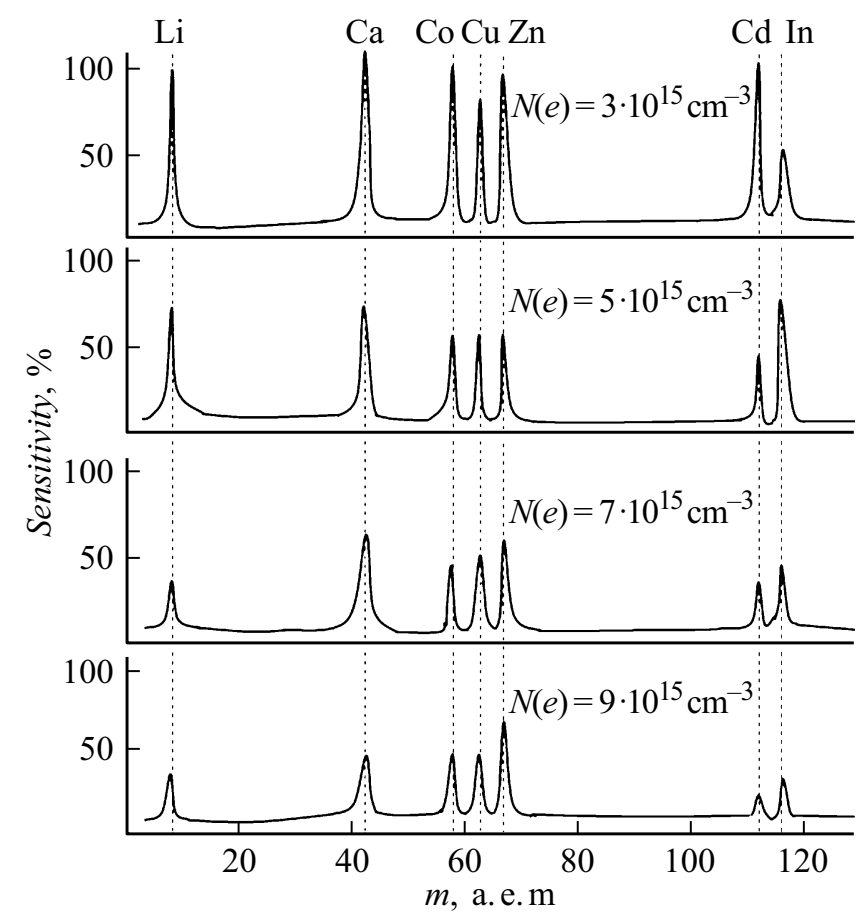

Рис. 4. Зависимость чувствительности анализатора от массы атомов элементов примесей для различных концентраций электронов при $T=7500 \mathrm{~K}$ и скорости распылительного аргона $v=12.01 / \mathrm{min}$.

атомной массы матричного элемента объясняется наличием других факторов, влияющих на чувствительность. Например, различие первых потенциалов ионизации разных элементов приводит к неодинаковому их влиянию на температуру плазмы в центральном канале [13].

На рис. 3 показана зависимость предельного значения концентрации электронов от скорости аргона для разных мощностей генератора ВЧ энергии. Видно, что уменьшение скорости потока через распылитель приводит к уменьшению концентрации электронов, что, в свою очередь, приводит к уменьшению подавления интенсивности сигнала на выходе (т.е. к увеличению чувствительности). Действительно, при уменьшении скорости аргона уменьшается плотность матричных ионов. По этой причине температура плазмы и время нахождения проб в центральной зоне увеличивается, это способствует более эффективной рекомбинации ионов аналита, следовательно, уменьшению интенсивности сигнала на выходе из анализатора.

Как уже было отмечено, степень подавления $\gamma\left(A_{n}\right)_{M_{t}}$ представляется многопараметрической функцией, поскольку степень ионизации атомов определяется в основном $\varphi_{i}^{+}\left(A_{n}\right), T$ и $N(e)$. Величина $\varphi^{+}\left(A_{n}\right)$ является справочной и ее воздействие на $K_{T}\left(A_{n}\right)$ рассчитывается. Температура разряда зависит от плазмообразующего газа и его расхода, от количества вводимого в плазму аэрозоля анализируемого раствора, а также от вкладываемой мощности высокочастотного генератора [15]. Эти параметры, как правило, фиксированы в многочисленных экспериментах. Температура в ICP довольно устойчива в больших диапазонах концентрации матричного элемента (особенно легкоионизируемых элементов [16]). Наиболее сложно проследить влияние $N(e)$ на значение $\gamma\left(A_{n}\right)_{M_{t}}$, поскольку концентрация электронов зависит от множества экспериментальных факторов при введении матричного элемента.

На рис. 4 и 5 приведено семейство масс-спектров элементов примеси $(m / q)$ для четырех значений концентрации электронов. Как видно из хода кривых, с увеличение концентрации электронов изменяется спектр масс, а именно амплитуды сигналов элементов более тяжелых элементов $(\mathrm{Cd}, \mathrm{In}, \mathrm{Ba}, \mathrm{Pb})$ подавляются заметнее, чем легких элементов ( $\mathrm{Li}, \mathrm{Ca}, \mathrm{Co}, \mathrm{Ni}, \mathrm{Cu}, \mathrm{Sr})$.

Эксперименты показывают, что в отсутствие матричных элементов при конкретной температуре плотность электронов определяется степенью ионизации аргона, которая, в свою очередь, является функцией мощности ВЧ генератора (рис. 4, 5).

При поступлении в плазму более тяжелых матричных элементов с первыми потенциалами ионизации ниже, чем у аргона, кислорода и водорода, изменяется ионизационный процесс, и в системе увеличивается концентрация электронов в центральной зоне. Таким образом, значение $N(e)$ в существенной мере определяется температурой разряда, потенциалом ионизации матричных элементов и исходным соотношением концентраций матричных элементов в плазме разряда.

Что касается роли повышенной концентрации электронов в подавлении аналитического ионного сигнала,

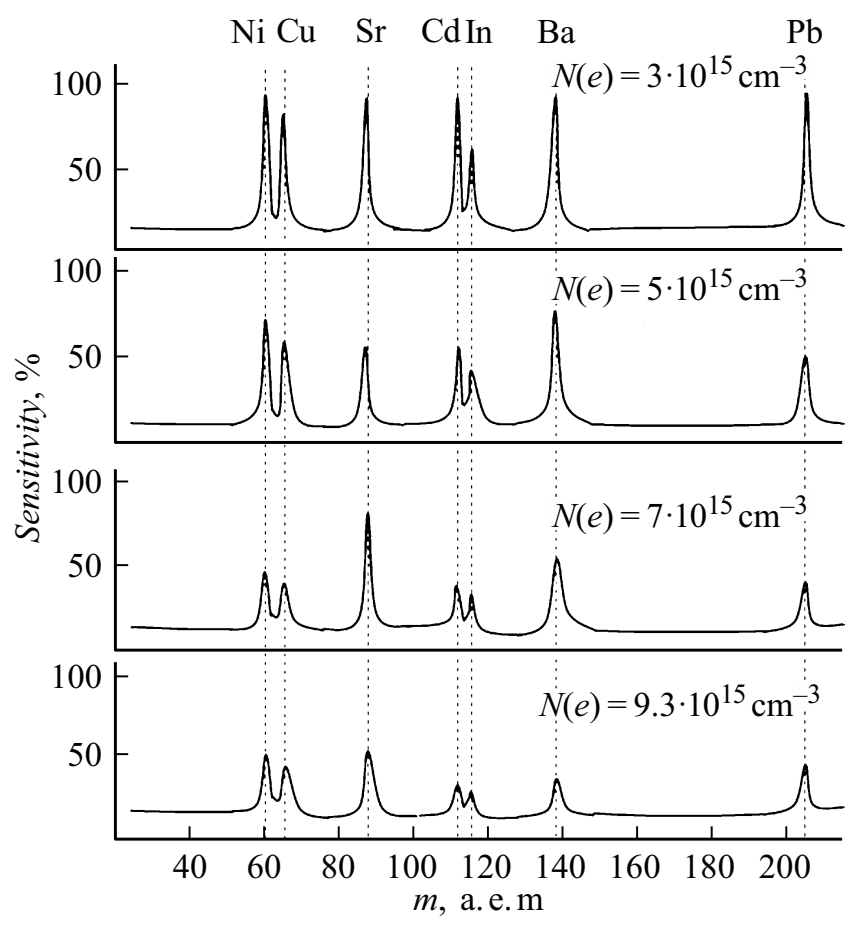

Рис. 5. Зависимость чувствительности анализатора от массы атомов элементов примесей при $T=7500 \mathrm{~K}$ и мощности ВЧ генератора $W=1450 \mathrm{~W}$. 
то она заключается, по-видимому, в интенсивной рекомбинации свободных электронов с ионами как аргоновой плазмы, так и ионами элементов анализируемого вещества. При этом чем выше плотность электронов, тем вероятнее рекомбинация. В работе [17] теоретически доказано, что высокая электронная плотность обеспечивается ионизацией атомов аргона и атомных компонентов гомогенных солей $\mathrm{NaCl}, \mathrm{MgCl}_{2}, \mathrm{NH}_{4} \mathrm{~J}$, вводимых с водным аэрозолем анализируемого раствора в различных концентрациях в плазму разряда.

Самой простой трактовкой подавления сигналов аналитов с относительно высокими $\varphi^{+}\left(A_{n}\right)$ является подавление их ионизации в растворах, содержащих соли легкоионизируемых элементов. Это происходит за счет увеличения эффективности ион-электронной рекомбинации при повышении плотности плазмы $[5,18,19]$.

\section{Заключение}

Таким образом, можно заключить, что неспектральный матричный эффект довольно сложен по своей природе. Многообразны не только варианты влияния даже одного вида помех, но и возможны различные механизмы, объясняющие наблюдаемые закономерности. Все приводит к тому, что, несмотря на более чем сорокалетнюю историю экспериментального и теоретического изучения проблемы, до сих пор трудно предсказать глубину влияния того или иного компонента матрицы на результаты определения, и полностью устранить это влияние в ходе анализа возможно только лишь инструментальными способами.

\section{Конфликт интересов}

Авторы заявляют, что у них нет конфликта интересов.

\section{Список литературы}

[1] E.H. Evans, J.J. Giglio. J. Anal. At. Spectrom., 8 (1), 1 (1993).

[2] N. Jakubowski, T. Prohaska, F. Vanhaecke, P.H. Roos, T. Lindemann. J. Anal. At. Spectrom., 26 (4), 727 (2011).

[3] C. Agatemor, D. Beauchemin. Anal. Chim. Acta, 706 (1), 66 (2011).

[4] E.R. Denoyer, D. Jacques, E. Debrach, S.D. Tanner. Atom. Spectrosc., 16, 1 (1995).

[5] А.Н. Зайдель, В.К. Прокофьев, С.М. Райский, В.А. Славный, Е.Я.Шрейдер. Таблицы спектральных линий. (Наука, М., 1969).

[6] R.S. Houk. Analytical Chemistry, 58, 97A (1986).

[7] J.A. Olivares, R.S. Houk. Anal. Chem., 58 (1), 20 (1986).

[8] J.R. Garbarino, H.E. Taylor. Anal. Chem., 59, 1568 (1987).

[9] А.А. Пупышев, А.К. Луцак, В.Н. Музгин. ЖАХ, 53 (7), 713 (1998).

[10] Т.К. Нурубейли. Электронная обработка материалов, $\mathbf{5 3}(4), 53$ (2017).
[11] Г.И. Беков, А.А. Бойцов, М.А. Большов, Е.Л. Гринзайд, А.И. Дробышев, Х.И. Зильберштейн, Д.А. Кацков, А.А. Петров, С.В. Подмошенская, Е.Д. Прудников, Б.Я. Юфа. Спектральный анализ чистых веществ. Под ред. Х. И. Зильберштейна. 2-е изд. (Химия, СПб., 1994).

[12] Т.К. Нурубейли. Проблемы энергетики, 1, 19 (2018).

[13] В.К. Карандашев, А.Ю. Лейкин, К.В. Жерноклеева. ЖАХ, 69 (1), 26 (2014).

[14] Плазма в лазерах. Ред. Дж. Бекефи. (Энергоиздат, М., 1986).

[15] А.А. Пупышев, А.К. Луцак, В.Н. Музгин. ЖАХ, 53, 713 (1990).

[16] К.П. Балуда, Л.Н. Филимонов, В.В. Недлер. Высокочастотный ИСП разряд в эмиссионном спектральном анализе. (Наука, Л., 1988).

[17] А.А. Пупышев, А.К. Луцак. ЖАХ, 53 (11), 1141 (1998).

[18] В.К. Карандашев, А.Н. Туранов, Т.А. Орлова, А.Е.Лежнев, С.В. Носенко, Н.И. Золотарева, И.Р. Москвина. Заводская лаборатория. Диагностика материалов, 73 (1), 12 (2007).

[19] M.M. Fraser, D. Beauchemin. Spectrochim Acta, Part B, 55 (11), 1705 (2000). 